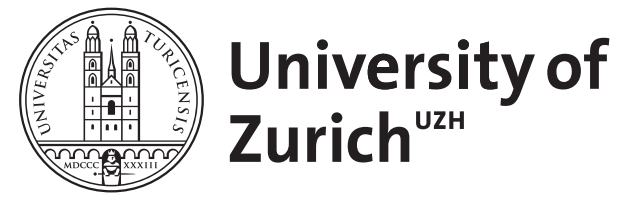

\title{
Der „Zusammenhang zwischen Natur- und Geisteswissenschaft" bei Aby Warburg, Edgar Wind und Niels Bohr
}

\author{
Targia, Giovanna
}

DOI: https://doi.org/10.1515/9783110583410-003

Posted at the Zurich Open Repository and Archive, University of Zurich ZORA URL: https://doi.org/10.5167/uzh-182985

Book Section

Published Version

Originally published at:

Targia, Giovanna (2019). Der „Zusammenhang zwischen Natur- und Geisteswissenschaft" bei Aby Warburg, Edgar Wind und Niels Bohr. In: Fehrenbach, Frank; Zumbusch, Cornelia. Aby Warburg und die Natur : Epistemik, Ästhetik, Kulturtheorie. Berlin: De Gruyter, 9-31.

DOI: https://doi.org/10.1515/9783110583410-003 
wohl einer gewissen Kurzsichtigkeit, einer radikal reduktionistischen Auffassung Vorschub geleistet, statt gerade das zu befördern, was Kandel auch im Rückblick nochmals ausdrücklich mit Freud in Verbindung gebracht hat, dass ein Verständnis neurophysiologischer Vorgänge uns tiefer in das allgemeine Verständnis der Kultur, nicht nur auf die Schliche der Zellbiologie leitet.

\section{Giovanna Targia}

\section{Der „Zusammenhang zwischen Natur- \\ und Geisteswissenschaft" bei Aby Warburg,}

\section{Edgar Wind und Niels Bohr}

\begin{abstract}
„Die Bedeutung der physikalischen Wissenschaft für das allgemeine philosophische Denken liegt nicht allein in ihrem Beitrag zu unserer stetig wachsenden Kenntnis der Natur, derer wir selbst ein Teil sind, sondern ebenso sehr darin, daß sie immer wieder zu einer Überprüfung und Verfeinerung unserer begrifflichen Hilfsmittel Anstoß gegeben hat."
\end{abstract}

Niels Bohr'

Der Zusammenhang zwischen Natur- und Geisteswissenschaften an der Bibliothek Warburg

In einem auf den 22. Juli 1927 datierten Eintrag im kollektiven Tagebuch seiner Bibliothek schreibt Aby Warburg, er wolle „den Zusammenhang zwischen Naturwissenschaft und Geisteswissenschaft" demonstrieren, indem er in seiner „Werkstatt [...] an der ,biologischen Psychologie des antikisierenden Dynamogramms' die Einheitlichkeit aller geistigen Orientierungsversuche" erfahren lasse.

Dieser gleichsam beiläufig erwähnte, in wissenschaftlicher Hinsicht jedoch zentrale Zusammenhang zwischen Natur- und Geisteswissenschaft erweist sich bei näherer Betrachtung als eine ständige Inspirationsquelle für Warburg, wie bereits seine eigenwilligen Begriffsübernahmen bzw. Wortprägungen zeigen. ${ }^{3}$ Daher scheint es angebracht, diese Inspirationsquelle und Warburgs Sprachgebrauch auch im Licht der jüngst publizierten Schriften aus dem Nachlass des Hamburger Kunst- und Kulturhistorikers zu analysieren. Inwieweit kann Warburg wegen seines Rekurses auf die naturwissenschaftliche

Niels Bohr: Atomphysik und menschliche Erkenntnis, Braunschweig 1958a, S.1.

Aby Warburg: Tagebuch der Kulturwissenschaftlichen Bibliothek Warburg [1926-1929] (Gesammelte Schriften Bd. VII), hg. von Karen Michels und Charlotte Schoell-Glass, Berlin 2001, S. 123.

3 Vgl. dazu beispielsweise Thomas Hensel: Aalsuppenstyl oder Metaphorologie? Zu Aby Warburgs Sprach- und Denkfiguren, in: Martin Treml, Sabine Flach und Pablo Schneider (Hg.): Warburgs Denkraum. Formen, Motive, Materialien, München 2014, S. 71-90. 
Terminologie für ein Kind des neunzehnten Jahrhunderts gehalten werden, und inwieweit charákterisiert eben diese Terminologie und Warburgs Sprachgebrauch die Besonderheit seines Forschungsansatzes? ${ }^{3}$ Welche sind die jeweiligen Stichwortgeber für seinen Begriffsund Sprachgebrauch und welche inhaltliche Wandlung erfahren diese Stichworte in Warburgs Anwendung? Wurde die Naturwissenschaft etwa auch zu einer Inspirationsquelle für Warburgs geistige Nachfolger?

Um diesen Fragen nachzugehen, bietet der anfangs zitierte Tagebucheintrag einen günstigen Ausgangspunkt. Er gehört einer entscheidenden, von Warburg selbst als „Heuernte bei Gewitter" definierten Phase seines Lebens und seiner wissenschaftlichen Tätigkeit an. ${ }^{5}$ Im Sommer 1927, ein Jahr nach der Eröffnung des neuen Gebäudes, das die Kulturwissenschaftliche Bibliothek bis Dezember 1933 beherbergen sollte, hatte der Hamburger Gelehrte ein Seminar über Jacob Burckhardt und die kulturwissenschaftliche Methode gehalten. ${ }^{6}$ Parallel dazu setzte er die Idee des Bilderatlas probeweise konkret durch Ausstellungen sowie durch neue Materialsammlungen und -anordnungen um. ${ }^{7}$ Am 16. Juli 1927, aus Anlass eines Vortrags gehalten von Erich Rothacker, ${ }^{8}$ hatte Warburg zwei „Bildertafeln" ausgestellt und vor einem ausgewählten Publikum kommentiert; die Bilderreihe trug den Titel Die Funktion der sozialen Mneme als Bewahrerin der antikisierenden Dynamo-Engramme der Gebärdensprache und illustrierte die antiken Wurzeln von Symbolen, die sich in der politischen Ikonographie bis in die Gegenwart bewahrt hatten: Fortuna, Kairos, Neptun, und der Löwe als Herrscherattribute. Es handelt sich wahrscheinlich um dasselbe Bildmaterial, das Warburg entsprechend dem oben genannten Tagebucheintrag vom 22. Juli für eine Darstellung des Zusammenhangs zwischen den Natur- und Geisteswissenschaften vor seiner Tochter Frede und dem Ehepaar Neumeyer verwendet hatte."

4 Vgl. Ernst Gombrich: Aby Warburg. An Intellectual Biography, London 1970, bes. S. 67-85; Ernst Gombrich: Warburg und der Evolutionismus des 19. Jahrhunderts, in: Robert Galitz und Brita Reimers (Hg.): Aby M. Warburg: „Ekstatische Nymphe ... trauernder Flussgott". Portrait eines Gelehrten, Hamburg 1995, S. 52-73 sowie Gombrichs fragmentarische Einleitung zu der geplanten Ausgabe des Bilderatlas Mnemosyne von 1939, zit. in Katia Mazzucco: The Work of Ernst H. Gombrich on the Aby M. Warburg Fragments, in: Journal of Art Historiography 5 (2011) S. 1-26, hier S. $19-20$.

5 Zur letzten Arbeitsphase Warburgs nach dessen Aufenthalt im Kreuzlinger Sanatorium vgl. Claudia Naber: „Heuernte bei Gewitter“: Aby Warburg 1924-1929, in: GALITZ UND ReIMERS 1995, S. 104-129.

6 Aby Warburg: Ausgewählte Schriften und Würdigungen, hg. von Dieter Wuttke, Baden-Baden 1992, S. 592, Nr. 99 und Bernd Roeck: Aby Warburgs Seminarübungen über Jacob Burckhardt im Sommersemester 1927, in: Idea. Jahrbuch der Hamburger Kunsthalle 10 (1991), S. 65-89.

7 Für die Chronologie der Arbeit am Bilderatlas vgl. Roland Recht: Introduction, in: Aby Warburg: L'Atlas Mnemosyne, Paris 2012, S. 7-48.

8 Näheres über den Besuch Rothackers in der KBW findet sich in: WARBURG [1926-1929] 2001, S. 119-123, sowie in Aby Warburg: Bilderreihen und Ausstellungen (Gesammelte Schriften Bd. II.2), hg. von Uwe Fleckner und Isabella Woldt, Berlin 2012, S. 135-140.

9 Vgl. WARBURG [1926-1929] 2001, S. 123.
Diese Notiz fasst grundlegende Aspekte von Warburgs Arbeitsweise zusammen: Von der Idee eines fruchtbaren Methodenaustauschs zwischen scheinbar gänzlich unterschiedlichen Disziplinen bis hin zur Auffassung seiner Bibliothek als einer offenen Werkstatt bzw. ein Laboratorium, ${ }^{10}$ welches, obwohl es die persönliche Gedankendisposition Warburgs reflektiert, sich auch für Mitarbeiter und Besucher als ein Ort der Gewinnung neuer Erkenntnisse eignete; von dem Konzept einer sowohl in der Biologie als auch in der Philologie wurzelnden Kulturwissenschaft bis hin zur Hypothese einer „Einheitlichkeit aller geistigen Orientierungsversuche" der Menschheit. Dieser letzte Punkt wurde bekanntlich zeitgleich auch durch Ernst Cassirer, einen in diesen Jahren ständigen Gesprächspartner Warburgs, innerhalb des Systems der "symbolischen Formen" erarbeitet." Warburgs Arbeitsweise weist auf einen der positivistisch orientierten kunsthistorischen Praxis gegenüber komplexeren Forschungsansatz hin: ${ }^{12}$ Der vermeintlichen naturwissenschaftlichen Exaktheit zog Warburg die Verortung der Kunstgeschichte innerhalb einer umfassenden Kulturwissenschaft vor, in der es keine hierarchische Gewichtung von unterschiedlichen „Orientierungsversuchen" gab.

Analog zu diesem Forschungsverständnis setzte Cassirer im 1939 publizierten Beitrag Naturalistische und humanistische Begründung der Kulturphilosophie den Akzent auf ebenjenes einheitliche Substrat der verschiedenen „Objektivierungsformen" von menschlichen Kultur. Indem der Philosoph die Krise des Kausalitätsbegriffs in der modernen Physik diskutierte, wies er auf Hermann von Helmholtz hin ${ }^{13}$ und nannte als gemeinsames Ziel

10 Vgl. Aby Warburg: Vom Arsenal zum Laboratorium [1927a], in: ders.: Werke in einem Band, hg. von Martin Treml, Sigrid Weigel und Perdita Ladwig, Berlin 2010, S. 683-694. Vgl. auch Peter van Huisstede: Der Mnemosyne-Atlas. Ein Laboratorium der Bildgeschichte, in: Gai.JTZ Und REIMERS 1995, S. 130-17l. Zu dem Vorstellungskreis vom, Labor' und ,Werkstatt' in den Geisteswissenschaften sei hier beispielsweise auf die Beobachtungen von Ulrich Raulff: Ein Historiker im 20. Jahrhundert: Marc Bloch, Frankfurt am Main 1995, S. 66-70 hingewiesen.

11 Über die zentrale Rolle der intellektuellen Beziehung zwischen Warburg und Cassirer sei hier zumindest hingewiesen auf: Claudia Naber: „....die Fackel deutsch-jüdischer Geistigkeit weitertragen". Der Hamburger Kreis um Ernst Cassirer und Aby Warburg in: Arno Herzig ( $\mathrm{Hg}$ ): Die Juden in Ho Jude in " Hanbus Formgebung. Ernst Cassirer und die Bibliothek Warburg, in. Vortage aus dem Warburg-Haus 1 (1997), S. 1-29; Massimo Ferrari: Ennst Cassirer. Dalla Scuola di Marburgo alla filosofia della cultura, Firenze 1996, S. 215-254; Cornelia Zumbusch: Wissenschaft in Bildern. Symbol und dialektisches Bild in Aby Warburgs Mnemosyne-Atlas und Walter Benjamins Passagen-Werk, Berlin 2004, bes. S. 229-246; Arno Schubbach: Das Zur-Erscheinung-Kommen des Menschen. Cassirers und Warburgs kulturphilosophische Anthropologie, in: Zeitschrift für Ästhetik und allgemeine Kunstwissenschaft, Sonderheft 14 (2016), S. 169-191; sowie auf die Beiträge in Giulio Raio (Hg.): Philosophy and Iconology. Cassirer Studies, Napoli 2008.

12 Vgl. z. B. Jutta Müller-Tamm, Henning Schmidgen und Tobias Wilke ( $\mathrm{Hg}$.): Gefühl und Genauigkeit. Empirische Âsthetik um 1900, München 2014 und Mitchell B. Frank und Daniel Adler ( $\mathrm{Hg}$ ) German Art History and Scientific Thought: Beyond Formalism, Farnham 2012.

13 Cassirer bezieht sich insbesondere auf Hermann von Helmboltz: Die Tatsachen in der Wahrnehmung [1878], in: ders.: Vorträge und Reden, Bd. 2, Braunschweig 1896, S. 213-247, hier S. 244. 
der Kulturphilosophie das „Verständnis der universellen Prinzipien der ,Formgebung überhaupt“. „Aber auch sie $e^{\text {“ }}$ - d. i. die Kulturphilosophie, fuhr Cassirer fort - „kann nicht prophezeien; am wenigsten dort, wo es sich nicht nur um physisches Sein und Geschehen, sondern um menschliches Tun handelt. Das Tun [...] ist nicht von vornherein an einen bestimmten, klar abzugrenzenden Kreis von Möglichkeiten gebunden, sondern muß sich ständig neue Möglichkeiten suchen und schaffen ". ${ }^{14}$ Daraus ergeben sich mindestens zwei Folgerungen: erstens die Zentralität ,unserer eigenen, subjektiven Verantwortung " ${ }^{15}$ und zweitens das Bewusstsein, dass wir ständig wissenschaftliche Verfahren entwickeln und verfeinern, die eine universelle Tragweite haben, und die zu kognitiven Hilfsmitteln werden können.

In Warburgs charakteristischer Arbeitsweise sowie in dessen individuellem Sprachgebrauch lässt sich gleichsam die konkrete Anwendung dieser Überlegungen beobachten. In dem anfangs zitierten Tagebucheintrag legt Warburg seine Arbeitshypothesen dar, indem er seine Argumentation folgendermaßen zusammenfasst: „ICh ging von Darwin und Piderit aus und suchte die Funktion des Gesetzes [des] kleinsten Kraftmaaßes aus der mnemischen Dauer (durch die Geschichte) der Engramme höchster Ergriffenheit (die ,Antike ist ein solcher Conservator) darzustellen. Da hierzu eine Bilderwand (von der Fortuna, über Neptun zur Briefmarke von Barbados) nötig war, mußte ich Saxl um Hilfe bitten $[\ldots]^{\text {«. }}, 16$

Entsprechend einer oft zitierten, autobiographischen Notiz desselben Jahres $1927^{17}$ erinnert Warburg hier erneut an die Lektüre, die für seine frühe Forschung in entscheidender Weise ausschlaggebend war. Aus den intellektuellen Anregungen, die Warburg aus den Schriften von Charles Darwin und Theodor Piderit erhielt - d.h. vielmehr aus der Geschichte der Biologie als der Kunstgeschichte - geht der Ansatz für eine Art Theorem hervor, das Warburg anhand von bildlichem Material zu beweisen versucht.

Warburg führt die Namen von Darwin und Piderit nicht etwa im Zusammenhang mit der Vergegenwärtigung bestimmter Resultate der experimentellen Psychologie auf, sondern vielmehr in Annäherung an die generelle Frage, auf welche Weise menschlicher Ausdruck in Bildern dargestellt werden könne. Eine biologische und phylogenetische Erklärung scheint für Warburg am überzeugendsten zu sein: Von Darwin übernimmt er die Auffassung der Ausdrucksbewegungen als fossiles Ưberbleibsel eines ursprünglichen, tierischen Verhaltens, die „Theorie von der Erinnerung an den sinnfälligen Reizzustand“18 und vor allem die Beobachtungen über die Ambivalenz des Ausdrucks extremer emotiver

14 Ernst Cassirer: Naturalistische und humanistische Begründung der Kulturphilosophie [1939], in ders.: Gesammelte Werke. Hamburger Ausgabe, Bd. 22: Aufsätze und kleine Schriften (1936-1940) hg. von Birgit Recki, Text und Anm, bearb. von Claus Rosenkranz, Hamburg 2006, S. 140-166, hier S. 165.

15 Cassirer [1939] 2006, S. 165.

16 WARBURG [1926-1929] 2001, S. 123

17 WARBURG [1927a] 2010 S. $687-688$

18 WARBURG $[1927$ a] 2010, S. 688.
Zustände. ${ }^{19}$ Vom Arzt und Schriftsteller Theodor Piderit andererseits rezipierte Warburg die Forschungen im Bereich der physiologischen Psychologie, gesammelt unter anderem in einer Monographie zur Mimik und Physiognomik, ${ }^{20}$ in der verschiedene Ausdrucksweisen des Seelenlebens im menschlichen Antlitz untersucht und eine Analyse von transitorischen, mimischen Zügen und der durch häufige Wiederholung bleibenden physiognomischen Eigenschaften veranschaulicht wurde. Darwins Thesen und Piderits Prinzipien boten Warburg die Basis für einen einheitlichen Erklärungsansatz spezifischer Ausdrucksphänomene als Gegenstand der bildenden Kunst.

Im Rahmen dieser philosophisch-naturwissenschaftlichen Prolegomena analysiert Warburg das Bildmaterial in historischen Zusammenhängen: Die in Bildern dargestellten Ausdrucksbewegungen haben sich in einer breiten Zeitspanne zu einem wirkungsvollen Repertoire künstlerischer Motive etabliert. Auch für diesen Prozess sucht Warburg eine Erklärung im Bereich der „biologischen Psychologie“, ${ }^{21}$ indem er anstrebt, die Dynamik unterschiedlicher Überlieferungsmöglichkeiten zu erschließen, nämlich der direkten, formalen und ikonographischen Überlieferung und der indirekten, über den „Austausch von Ausdruckswerten ${ }^{{ }_{22} 2}$ vermittelten Übertragung und Anpassung von „Formeln“ an die jeweiligen kulturellen Kontexte.

Um schließlich seine These zu formulieren, bedient sich Warburg, neben den Begriffen „mnemisch“ und „Engramm “ - bekanntermaßen abgeleitet von der zeitgenössischen, physio-psychologischen Forschung über das Gedächtnis ${ }^{23}$ - auch des „Gesetz[es] des kleinsten Kraftmaaßes". Dieses Prinzip - bereits im Konvolut der sogenannten Fragmente zur Ausdruckskunde (1888-1905) erwähnt ${ }^{24}$ - ist vom denkökonomischen Ansatz Richard

19 Zu Warburgs Darwin-Lektüre vgl. Carlo Ginzburg: Le forbici di Warburg, in: Maria Luisa Catoni (Hg.): Tre figure: Achille, Meleagro, Cristo, Milano 2013, S. 109-132, hier S. 114-117.

20 Theodor Piderit: Mimik und Physiognomik, Detmold 1886. Theoretische Grundsätze im Bereich der Physiologie und Psychologie werden hier neben ihren praktischen Gebrauch von Künstlern und Kunstkritikern in Betracht gezogen. Vgl. Georg Braungart: Leibhafter Sinn. Der andere Diskurs der Moderne, Tübingen 1995, bes. S. 156-172.

21 WARBURG [1926-1929] 2001, S. 123 .

22 Aby Warburg: Mnemosyne Binleitung [1929a], in: ders. Werke in einem Band, ho von Martin Treml, Sigrid Weigel und Perdita Ladwig, Berlin 2010, S. 629-639, hier S. 636

23 Ewald Hering: Über das Gedächtnis als eine allgemeine Funktion der organisierten Materie. Vortrag gehalten in der feierlichen Sitzung der Kaiserlichen Akademie der Wissenschaften in Wien am 30. Mai 1870, Leipzig 1905, und Richard Semon: Die Mneme als erhaltendes Prinzip im Wechsel des organischen Geschehens [1906], 3., stark umgearbeitete Aufl., Leipzig 1911. Vgl. dazu beispielsweise Stefan Rieger: Richard Semon und/oder Aby Warburg: Mneme und/oder Mnemosyne, in: Deutsche Vierteljahresschrift für Literaturwissenschaft und Geistesgeschichte 72 (1998), Sonderheft Medien des Gedächtnisses, S. 245-263 und Andrea Pinotti: Materia è memoria. Aby Warburg e le teorie della Mneme, in: C. Cieri Via und P. Montani (Hg.): Lo sguardo di Giano, Turin 2004, S. 53-78.

24 Vgl. z. B. Aby Warburg: Frammenti sull'espressione (Grundlegende Bruchstücke zu einer pragmatischen Ausdruckskunde), hg von Susanne Müller, Pisa 2011, S. 93, S. 94, S. 102, S. 103, S. 111 S. 124. Es handelt sich hier um Aphorismen aus den Jahren 1892-1897, wo das Prinzip anch in der uch in der entsprechenden, entgegengesetzten Variante als "Gesetz des größten Kraftmaßes" auftaucht. Wie 
Avenarius' sowie von der Arbeit Ernst Machs über die Analyse der Empfindungen ${ }^{25}$ abgeleitet: Die lex parsimoniae naturae wurde als Erklärungsmodell für die kulturelle Ưberlieferung zu einer lex parsimoniae mentis erweitert bzw. in eine solche überführt, und bildete somit für Warburg eine Grundlage zur demonstrativen Anordnung des kunsthistorischen Materials auf seinen Bilderwänden. Beispielsweise behält in diesem Sinne die antike Figur der Fortuna, die in semantischer Hinsicht in Verbindung mit einer mit dem Meeresgot Neptun assoziierten, naturhaften Sphäre steht, bis zum heutigen Tage einen schicksalhaf ten Charakter, wenn sie in Darstellungen weltlicher Herrscher vorkommt. Hier lässt sich eine longue durée der bildlichen und symbolischen Überlieferung vernehmen, die in physikalischen Termini als ,Beharrung' beschrieben werden kann und gemäß dem „Gesetz des kleinsten Kraftmaaßes" wirkt: auf einer Briefmarke von Barbados (1903-1910) erschein König Eduard VII. (1841-1910) als Neptun, Herrscher der Meere mit ihrer Unvorhersehbarkeit und ihrer ehrfurchterregenden Weite.

Es war wiederum Ernst Cassirer, der diese ,Beharrung als zentralen Punkt evozierte, als er in einem Aufsatz der frühen vierziger Jahre auf Warburgs Arbeitsweise hinwies:

„Die Formsprache gewinnt eine solche Festigkeit, daß bestimmte Themata mit bestimmten Weisen des Ausdrucks fest zu verwachsen scheinen, daß sie uns immer wieder in denselben oder leicht modifizierten Formen begegnen. Dieses ,Beharrungsgesetz', das für die Fortbewegung der Formen gilt, bildet einen der wichtigsten Faktoren der künstlerischen Entwicklung - und für die Kunstgeschichte liegt hier eine der reizvollsten Aufgaben. In neuerer Zeit ist es insbesondere Aby Warburg gewesen, der auf diesen Prozeß das stärkste Gewicht gelegt und der ihn, psychologisch wie historisch, nach allen Seiten hin zu erleuchten gesucht hat. Warburg ist ursprünglich von der Kunstgeschichte der italienischen Renaissance ausgegangen. Aber sie bildet für ihn nur ein einzelnes Paradigma, an dem er sich die Eigenart und die Grundrichtung des schöpferischen Prozesses in der bildenden Kunst klarmachen wollte. Beides

auch bei anderen Warburgschen Termini feststellbar (wie z. B. „Pathosformel“ oder „energetische Inversion"), sollte auch dieser zu einer Art technischen Wortschatz aufgenommen werden, der auch von Warbur Mitarbetern benutzt werden solte: Sieh z B. Whaf S. 342. V ] auch W T S. 342. Vgl. aUCh WARBURG [1927a] 2010, S. 688 mit Bezug auf Darwins „einfache Maxime de Ausdruckskunde - Reizerinnerung nach dem Gesetz des grössten Kraftmasses bei Gesichtsaus drucksbewegungen“, sowie folgende Stelle aus dem berühmten Kreuzlingen Vortrag: „Im Reich der Mythologik herrscht nicht das Gesetz vom kleinsten Kraftmass, es wird nicht nach dem kleinsten Erreger der Gesetzmässigkeit im naturgesetzlichen Ablauf gesucht, sondern es wird um der Fassbarkeit willen ein möglichst mit dämonischer Kraft gesättigtes Wesen eingesetzt, um die Ursache der rätselhaften Geschehnisse wirklich packen zu können“: WARBURG [1923a] 2010, S. 558.

25 Warburg besaß sowohl Richard Avenarius's Buch Philosophie als Denken der Welt gemäss dem Princip des kleinsten Kraftmasses. Prolegomena zu einer Kritik der reinen Erfahrung, Leipzig 1876 (in Warburgs Bibliothek mit der Signatur AMH 525 versehen) als auch Ernst Mach. Die Analyse der Empfindungen und das Vehistes des Physischen zum Psychischen 3, verm. Aut. fand er am deutlichsten ausgedrückt in dem Nachleben der antiken Bildformen. [...] Den Bestand und den Wandel, die Statik und die Dynamik dieser, Pathosformeln" hat Warburg durch die gesamte Geschichte der bildenden Kunst hindurch verfolg "“" ${ }^{26}$

Einerseits stellten also für Warburg die Bilder auf den Tafeln gleichsam die konkrete Exemplifikation eines, Beharrungsgesetzes ${ }^{\imath}$ dar, als wären sie in geduldiger Arbeit geknüpfte Figuren eines Teppichs; die Hinweise auf Darwin, Piderit, das, Gesetz des kleinsten Kraftmaßes', die Auffassung der Antike als, Conservator enthüllen andererseits das heuristische Prinzip, das die Hand des Teppichknüpfers und die Anordnung der Figuren angeführt hat: Warburgs Sprachgebrauch ermöglicht daher, gleichsam die Arbeitsweise von der Rückseite des Geknüpften her zu betrachten.

\section{Die Rückseite des Teppichs}

Ein enger Zusammenhang zwischen den publizierten Schriften Warburgs und seinen heuristischen Hypothesen lässt sich seit Beginn seiner Forschungstätigkeit beobachten. Die frühen Aphorismen mögen öfters kryptisch erscheinen, wenn sie von Warburgs historischen Untersuchungen isoliert werden. Bereits seine Dissertation über Botticelli war von Vier Thesen begleitet, die als Grundlage zu deren Verteidigung vorbereitet wurden: ${ }^{27}$ Diese zwei Texte bilden demnach ein einheitliches Ganzes, wobei die Vier Thesen als die Rückseite des Knüpfwerks betrachtet werden können, auf welchem der hinter der Beispielreihe liegende komplexe Gedankengang dargelegt wird.

Bekannterweise wird im Aufsatz über Botticelli eine umfassende Untersuchung der Literatur, der bildenden Kunst, des Theater- und des Festwesens um den Florentiner Künstler durchgeführt, die es sich zum Ziel setzt, möglichst fassbare Kriterien für die Definition des „Einflusses der Antike“ zu ermitteln. Warburg konnte die nachhaltige Verbreitung dieses Phänomens zeigen, indem er die einzelnen Übernahmen aus der Antike zu einer Formel vereinte: „die Behandlung des bewegten Beiwerks“ stellt für ihn das „Kriterium des ,Einflußes der Antike“" bzw. das „Merkmal der, antikisierenden Formgebung“ dar. ${ }^{28}$ Dieser Beschreibung entsprechen die synthetischeren Formeln, enthalten in den Vier Thesen,

26 Ernst Cassirer: Zur Logik der Kulturwissenschaften [1941], in: ders.: Gesammelte Werke. Hamburger Ausgabe, Bd. 24: Aufsätze und kleine Schriften (1941-1946), hg. von Birgit Recki, Text und Anm. bearb. von Claus Rosenkranz, Hamburg 2007, S. 355-486, hier S. 476.

27 Aby Warburg: Die Erneuerung der heidnischen Antike. Kulturwissenschaftliche Beiträge zur Geschichte der europäischen Renaissance, Gesammelte Schriften. Bd. I.1-2, hg. von Horst Bredekamp und Michael Diers, Leipzig u. a. 1998, S. 58 und Aby Warburg: Fragmente zur Ausdruckskunde (Gesammelte Schriften Bd. IV), hg. von Ulrich Pfisterer und Hans Christian Hönes, Berlin u. a. 2015, S. 285-294 sowie Warburg [1927a] 2010, S. 108-109 und S. 123.

28 WARBURG 1998, Bd. I.1, S. 19 und S. 30 . 
z. B.: Wenn eine „dynamisierende Zusatzform“ erscheint, ist sie das Zeichen eines antikisierenden Vorbilds. ${ }^{29}$

Zeitgleich zur Dissertation entstand außerdem der Text der chronologisch geordneten Fragmente zur Ausdruckskunde, wo eine frühe Akzentverschiebung spürbar ist: Ausgehend von beschreibenden Beobachtungen zu Realismus und Idealismus, ${ }^{30}$ dringt Warburg in die psychologischen Faktoren ein, die auf der Basis der künstlerischen Schöpfung und Rezeption stehen; mit der Zeit und erweiterten Lektüre tauchen auch allmählich physikalische Erklärungsansätze für die Auseinandersetzung dieser Faktoren auf. Der künstlerische Stil wird immer mehr als Resultat von komplexen Interaktionen auf unterschiedlichen Ebenen - auf der sozialen wie psychologischen, religionsgeschichtlichen wie rein künstlerischen Ebene - aufgefasst. ${ }^{31}$

In seiner Dissertation schreibt Warburg über Botticellis Geburt der Venus: „die blasenden Jünglingsköpfe, die der Maler anbringen soll, um die Bewegung in Haar und Gewandung zu ,begründen', sind ein rechtes Kompromißprodukt zwischen anthropomorphistischer Phantasie und vergleichender Reflexion “. ${ }^{32}$ Neben der Untersuchung über die naturalistische Kausalerklärung von Bewegungen in künstlerischen Darstellungen tritt hier die Idee des ,Kompromißprodukts ${ }^{c}$ auf, wobei in den Fragmenten solche Kompromiße als physikalische Gleichgewichtszustände betrachtet werden. In einem etwas späteren Fragment heißt es: „Es wäre das eigentliche Objekt der Kulturgeschichte, den jeweiligen Stand der Besonnenheit zu beschreiben ${ }^{c_{33}}$ - als wäre die Besonnenheit bzw. die „vergleichende Reflexion“ eine messbare Größe.

Es vollzieht sich hier also auch eine Akzentverschiebung vom Darstellungsgegenstand zum Darstellungsprozess, d.h. ein Übergang von der Betrachtung der Natur als dargestellter Gegenstand (also von der klassischen Frage der Naturwiedergabe) hin zur Betrachtung der Natur als schöpferische Kraft. Begriffliche Entlehnungen aus der Physik werden zu logischen Instrumenten, um eine Erklärung des schöpferischen Prozesses zu skizzieren.

29 Warburg 1998, Bd. I.1, S. 58 und WarbuRg 2015, S. 285-294. Diese als Schlussfolgerung ausgedrückte Formel könnte neben die berühmte Formel Jacob Burckhardts gestellt werden: Wo irgend Pathos zum Vorschein kam, mußte es in jener [i.e. antiker] Form geschehen" (Jacob Burcthhardt: Die Kultur der Renaissance in Italien: Ein Versuch [1860], in: ders. Werke. Kritische Gesamtaugabe, Bd. 4, hg. von Mikkel Mangold auf der Grundlage der Vorarbeiten von Kenji Hara und Hirouga-

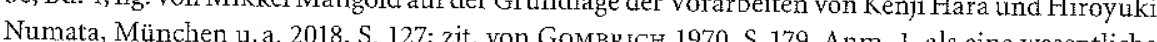
Referenz für Warburg).

Vgl. 7 B. einige frübs)

Vgl. z. B. einige frühe Fragmente, die unter dem Titel „Natur, Kunst und Geschichte" notiert sind: WARBURG 2011, S.31.

31 Vgl. WARBURG 2011, S. 41, S. 51, S. 65; vgl. dazu: Susanne Müller und Giovanna Targia: Die „Bruchstücke" Aby Warburgs und die Frage des Stils, in: Sabine Frommel und Antonio Brucculeri $(\mathrm{Hg}$.): L'idée du style dans l'historiographie artistique. Variantes nationales et transmissions, Rom 2012, S. $199-213$.

32 WARBURG 1998, Bd. I.1, S. 11-12.

33 Warburg 2011, S. 116
Man denke nur an die Anwendung des Begriffs „Kraft" und seiner Variationen: In den Fragmenten zur Ausdruckskunde spricht Warburg von der „Kraft der materialisierten Bewegung" ${ }^{34}$ wobei die dargesteliten in einer Bewegung verharrten Figuren in geometrischen und richtungsweisenden Begriffen mit den Worten, Vorwärts- bzw. Rückwärtsbewegung', ,Richtungsänderung', ,Kinetik', ,Lokomotion', ,Oscillation vom / des Ruhepunkt(es) $)^{c}$, ruhender' oder, dynamischer Zustand 'beschrieben sind.

Es handelt sich allerdings um eine Terminologie, die sich auch bei anderen Autoren wie etwa Gottfried Semper ${ }^{35}$ oder August Schmarsow, ${ }^{36}$ Warburgs Mentor während seines Florentiner Semesters 1888-1889, findet. Warburgs Anwendung dieser Begrifflichkeit scheint aber seinen eigentümlichen systematischen Versuch nachzuweisen, die psychophysiologische Theorie mit seinen schlüssigen philologischen Untersuchungen zu kombinieren: Die theoretische Dimension bleibt dabei immer auf der ,Rückseite des Teppichs', lenkt aber gleichzeitig die komplexe, historische Rekonstruktion, indem sie die begrifflichen Mittel bietet, um die unterschiedlichen historischen Fäden miteinander zu verknüpfen. Bedeutsam erscheint in dieser Hinsicht die Episode eines temporären Bruchs zwischen Warburg und Schmarsow nach der Erscheinung von Warburgs Dissertation: ${ }^{37}$ Schmarsow hatte Warburgs philologische Methode kritisiert, worauf Warburg mit zwei bitteren Briefen reagierte; erst 1907 war der Bruch überwunden, als sich Warburg mit einem Beitrag an der Festschrift für seinen ehemaligen Mentor beteiligte..$^{38}$

Eine weitere, berühmtere Episode beleuchtet das Verhältnis zwischen beiden Seiten des Teppichs näher. Auf das Jahr 1900 datiert der fiktive Briefwechsel zwischen Warburg

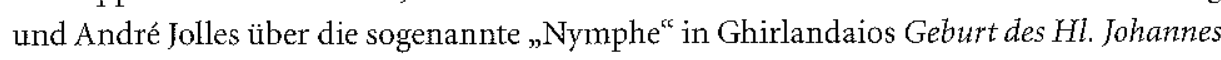
des Täufers im Chor von Santa Maria Novella in Florenz. ${ }^{39}$ Dieser romanhafte Lusus über die „Ninfa fiorentina" bildet ein literarisch anspruchsvolles a parte im Vergleich zu Warburgs ausdauernder Arbeit an den archivalischen Rekonstruktionen der Geschichte der Florentiner Frührenaissance sowie zu der entsprechenden, versuchsweisen theoretischen Systematisierung. Der erste fiktive Brief von Jolles an Warburg trägt das Datum 13. April 1900.

34 WARBURG 2011, S.92.

$35 \mathrm{Zu}$ Warburgs Lektüre von Semper vgl. Spyros Papapetros: Denkraum als Ornament. Warburg Semper und der kosmische Schmuck, in: TremL, FLACH und SChNeider 2014, S. 91-119.

36 Siehe z. B. August Schmarsow: Grundbegriffe der Kunstwissenschaft am Übergang vom Altertum zum Mittelalter kritisch erörtert und in systematischem Zusammenhange dargestellt, Leipzig u. a. 1905.

37 Vgl. die Briefe von Warburg an Schmarsow vom 15/07/1893 und 19/07/1893, in London, Warburg Institute Archive (WIA), General Correspondence (GC), in denen sich Warburg auf Schmarsows Artikel in der National-Zeitung vomn 11. Juli 1893 bezieht, in dem Einwände gegen Warburgs Methode und Resultate erhoben werden.

38 Vgl. den Brief von Warburg an Schmarsow, 24/07/1907, WIA, GC, zit. nach Spyros Papapetros: On the Animation of the Inorganic. Art, Architecture, and the Extension of Life, Chicago u.a. 2012, S. 324, Anm. 11.

39 Vgl. Aby Warburg: Ninfa Florentina [1900], in: ders.: Werke in einem Band, hg. von Martin Treml, Sigrid Weigel und Perdita Ladwig, Berlin 2010, S. 198-210. 
Wenige Tage später, am 23. April, verdichtet Warburg darauf hin seine Ideen zu Ghirlandaios Fresken in einer deutlich trockeneren Form: „Die angleichende [mimische] Richtungsbestimmung wird zur ausgleichenden [physiognomischen] Umfangsbestimmung.

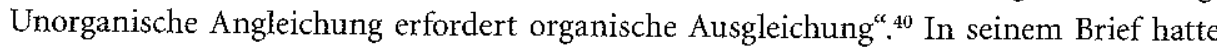
Jolles die „Nymphe“ als „die verkörperte Bewegung" definiert, worauf Warburg mit ähnlichen beschreibenden Ausdrücken geantwortet hatte; ${ }^{41}$ zeitgleïch jedoch rekurrierte War-

burg, in seinen Fragmenten zur Ausdruckskunde, auf geometrische und physikalische Begriffe, um die Antworten der Künstler auf die Frage nach der Darstellung von Bewegung - mit besonderer Berücksichtigung der Ornamente bzw. des "Beiwerks" - in der bildenden Kunst formelhaft darzustellen. In einer noch stärker verdichteten Ausdrucksweise sollte Warburg schließlich in den späten 1920er Jahren über „Dynamogramme“ bzw. „Dynamo-engramme" der Gebärdensprache schreiben.

In seinen frühen wie späten Arbeiten lässt sich aber eine stetige Verbindung zwischen historisch-philologischen Rekonstruktionen und heuristischen Hypothesen bzw. „allgemeinen Ideen" (d.h. zwischen der vorderen und rückseitigen Oberfläche des Teppichs) deutlich beobachten. Im fiktiven Briefwechsel mit Jolles nimmt Warburg die Rolle des Philologen ein, der auf die formalen Ahnen der „Nymphe" hinweist. In Bezug auf seine Methode schreibt er etwa am Schluss des Aufsatzes über Francesco Sassetti, dass die entscheidenden Widerstandsmomente organischer Stilentwicklung [...] uns erst durch die historisch-analytische Behandlung solcher Ausgleichsversuche klar" ${ }^{{ }^{42}}$ würden. Das physikalisch-mechanische Modell des Widerstands und Ausgleichs findet ständig seinen Kontrapunkt in der philologischen Forschung und wird dadurch überprüft.

\section{Symbolik und Schwerkraft}

Begriffliche Entlehnungen aus dem Bereich der Physik lassen sich auch auf einer weiteren Ebene von Warburgs Untersuchungen nachweisen. In den zahlreichen Fragmenten, die dem Problem des Symbols bzw. des Symbolisierens gewidmet sind, bezieht sich der Autor auf das Modell des Gravitationsgesetzes. Den Anstoß dazu gab ihm in diesem Fall eine Stelle in Heinrich von Steins Entstehung der neueren Aesthetik, ${ }^{43}$ in der konstatiert wird,

40 WARBURG 2011, Nr. 249 und 249a, S. 92-93.

41 Warburg [1900] 2010, S. 202.

42 WARBURg 1998, Bd. I.l. S. 158

43 Heinrich von Stein: Die Entstehung der neueren Aesthetik, Stuttgart 1886, S. 321, exzerpiert vo Warburg in WARBURG 2011, S. 142: „Mit dem einzig absolut Wichtigen und Werthvollen der ganzen Welt, nämlich der Eigenart des menschlichen Innern, darf man nicht wie mit den Buchstaben einer algebraischen Rechnung verfahren wollen. Ist man jedoch den Bewegungen des menschlichen Empfindens, das heisst also der ästhetischen Geschichte, in mancherlei Richtungen beobachtend nachgegangen: so darf dann angedeutet werden, dass hier Gesetze vorhanden zu sein scheinen, welche ebenso mächtig als die Gravitation, aber von der Uebertragungsform mechanischer Wirkungen verschieden sind - welche auch durch Analyse der Racen- und Machtverhältnisse der Menshenwelt sich nicht vollständig und ihrem wahren Wesen nach erkennen lassen". dass in der Geschichte der Ästhetik Gesetze vorhanden seien, die „ebenso mächtig als die Gravitation" sind. Warburg notierte sich die Stelle aus Steins Buch neben folgendem Fragment: „Physik des Denkens und Kunst. [...] Für die Physik des Denkens ist die Lehre von der Symbolik bzw. von der Kunst das [, ] was für die Kosmologie die Lehre von der Schwerkraft ist. Im realen Leben wirkt das Symbol als Anziehungskraft in der Ebene ${ }^{\text {“. }{ }^{44} \mathrm{Als}}$ Beispiel dafür führt hier Warburg einen Passus von einem seiner beliebtesten Autoren, Thomas Carlyle, an: das dritte Kapitel des dritten Buchs seines Sartor resartus ist den Symbolen gewidmet und erklärt den Unterschied zwischen ihrer „intrinsic and extrinsic meaning". Im Unterschied zur akzidentellen und rein äußerlichen Bedeutung der politischen oder sogar religiösen Symbole schreibt Carlyle dem "true Work of Art" eine ,innewohnende Bedeutung $\mathrm{zu}$, weil es "of itself fit that men should unite around $i^{\star 44}$ ist, als würde es eine magnetische Anziehungskraft ausüben. ${ }^{46}$

In die 1890er Jahre sind mehrere Fragmente datiert, in denen Warburgs Überlegungen rund um den Begriff „Erhaltung der Kraft ${ }^{{ }^{\alpha} 7}$ im Bezug auf das ,Beharrungsgesetz ${ }^{`}$ des Erinnerungsbildes kreisen.

In der Zeit zwischen der Erscheinung seiner Dissertation und diesen Aphorismen fallen die Impulse, die Warburg in seinem Berliner Semester durch das Studium der Psychologie bei Hermann Ebbinghaus sowie auf der amerikanischen Reise (September 1895 bis Juni 1896) erhalten hatte. Eine neue Terminologie taucht in den Fragmenten auf als eine Fortsetzung seiner Gedankenexperimente mit neuen Instrumenten: „Ganglien“, „Nerven“, „Reize“, „Vibration des ganzen Stranges“ . ${ }^{48}$ Die aus der Physik entlehnten Erklärungsmodelle werden trotzdem beibehalten. Der Begriff der „Energie“ ${ }^{\text {“ }}$. B. kommt seit

44 Warburg 2011, Nr. 403, S. 141. Vgl. Claudia Wedepohl: Pathos - Polarität - Distanz - Denkraum Eine archivalische Spurensuche, in: TremL, FLACH UND SCHNeIDER 2014, S. 17-49, bes. S. 25-28 und Thomas Schindler: Zwischen Empfinden und Denken. Aspekte zur Kulturpsychologie von Aby Warburg, Münster 2000, S. 83-89.

45 Vgl. Book III, chapter III („Symbols") in Thomas Carlyle: Sartor Resartus. The Life and Opinion of Herr Teufelsdröckh in three Books, Einl. und Anm. von Rodger L. Tarr, Textbearb. von Mark Engel und Rodger L. Tarr, London u. a. 2000, S. 161-166, hier S. 164-165.

46 Warburg nahm diesen Gedanken wieder auf und arbeitete bis zu seinen letzten Projekten an dieWen weiter; vgl. etwa den The sem weiter tivistisch zu betrachten. Einerseits haben sie eine Schwer der Praung die den Maximalwerten als intensiva und extensiva eine mnemische Dauer verbürgt. Andererseits sind diese Prägewerte ,gefühllose " Monaden ohne Fenstex, die erst durch Berührung mit dem selektiven Wollen der Epoche zu Funktionen der Anziehung oder Abstoßung von Leben werden": WARBURG [1926-1929] 2001, S. 339. Zum Besuch bei Einstein vgl. Horst Bredekamp und Claudia Wedepohl: Warburg, Cassirer und Einstein im Gespräch. Kepler als Schlüssel der Moderne, Berlin 2015, bes. S. 56-85.

47 Vgl. WarbuRg 2011, S. 104, Nr. 294 vom 23.VI.95: „Wir bezeichnen als besondere Leistungen der künstlerischen (Ein)bildungskraft die durch die Trägheit [Constanz] der Erinnerungsbilder hervorgerufene / sinnbildliche / typische / anthropomorphistische Zusatzform / Die subjektive Kraftersparniß äußert sich in objektiver Kraft (Raum, Zeit) Verschwendung., Erhaltung der Kraft"

48 Vgl. Zumbuscr 2004, S. 237, Anm. 21 über Warburgs Mitschrift zu den Psychologievorlesungen bei Ebbinghaus. 
1894 immer häufiger in den Fragmenten vor, und die in der Schrift über Botticelli dargelegte Argumentation wird von einem neuen Standpunkt aus neu überdacht. 1895 spricht Warburg von einer „Energie der Erinnerung ${ }^{449}$ und ein Jahr später, bei einer Überarbeitung einer der Vier Thesen, schreibt er: „die sonst zu neuer Differenzirung verwandte nervöse Energie [wird veranlaßt] zur Ausschaltung eines analogen intensiveren Erinnerungsreizes". ${ }^{\text {"0 }}$

Das ,energetische ${ }^{c}$ Modell stellt einen ständigen und suggestiven Bezugspunkt bis hin zu Warburgs späteren Arbeiten dar: man denke nur an den Ausdruck „energetische Inversion", verwendet für die Charakterisierung der Funktion der Pathosformeln, oder an die allgemeinere Beschreibung der „energetischen Umverseelung des dargestellten Menschentums “ in dem Aufsatz über Manets Déjeuner sur l'herbe. ${ }^{51}$ Im Juni 1927 zeigte der Autor in dem elliptischen Saal seiner Bibliothek eine Bilderreihe mit dem Titel Die Funktion der nachlebenden Antike bei der Ausprägung energetischer Symbolik, ${ }^{52}$ während Aufzeichnungen aus denselben Jahren in Notizheften und im Tagebuch der KBW eine Reihe von Arbeitstiteln und Untertiteln für das Projekt des Bilderatlasses dokumentieren: „das Erwachen der Heidengötter im Zeitalter der europäischen Renaissance als energetische Ausdruckswertbildung”; 53 "Transformatio energetica als Forschungsobjekt und Eigenfunktion einer vergleichend historischen Symbolbibliothek (das Symbol als katalytische Quintessenz)“; ${ }^{54}$ „Energetische Inversionen, Magnetisierung, auseinandertretende Pole: Ruhe (Andacht) plus flandrischem Andachtsbild und Bewegung (Triumph, antike Skulptur) bei Ghirlandajo ${ }^{\text {“. } 55}$

Bereits in seinen Untersuchungen zum Florentiner künstlerischen Umfeld um Lorenzo de' Medici in den Jahren 1901-1909 hatte Warburg die „Einwirkung der Umwelt

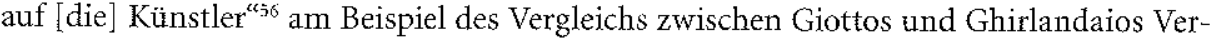
sionen der Bestätigung der Ordensregel des Hl. Franziskus durch Papst Innozenz III. analysiert und mit den Begriffen „Ausgleich" und „Schwingungsweite" gearbeitet. Am Anfang des Aufsatzes Bildniskunst und Florentiner Bürgertum nennt Warburg die ,entwickelnden

49 WARbURG 2011, S. 103, Nr. 290, 24.VI.1895. Vgl. Cornelia Zumbusch: Transformationen. Aby Warburg und die Kraft der Kunst, in: Frank Fehrenbach, Robert Felfe und Karin Leonhard $(\mathrm{Hg}$.$) :$ Kraft Intensität, Energie. Zur Dynanik der Kunst, Berlin u. a. 2018 S. 339-342; Gionand (H.): Energie der Umformung" als ,Reaktionsenergie". Aby Warburgs Vorträge über die Kultur der italienischen Frührenaissance (1909), in: Fehrenbach, Felfe und Leonhard 2018, S. 317-328.

50 WARBURG 201I, S. 113, Nr. 322, 2.VIII.1896.

1 Aby Warburg: Manet's Déjeuner sur l'herbe. Die vorprägende Funktion heidnischer Elementargottheiten für die Entwicklung modernen Naturgefühls [1929b], in: ders.: Werke in einem Band, hg. von Martin TremI, Sigrid Weigel und Perdita Ladwig, Berlin 2010, S. 647-659, hier S. 648.

52 WARBURG 2012, S. 115-133.

53 Warburgi [1927-1929] 2010, S. 643 .

54 WARBURG [1927-1929] 2010, S. 644

55 WARBURG [1927-1929] 2010, S. 646

56 WARBURG 1998, Bd. I.1, S. 96 .
Kräfte einer lebendigen Porträtkunst ${ }^{457}$ im Hinblick auf die Beziehungen zwischen Künstlern und Auftraggebern sowie auf die besonderen, historischen und kulturellen Verhältnisse der untersuchten Epoche. Er bezeichnet demnach die Kunstwerke der Florentiner Frührenaissance „von vornherein gewissermaßen als Ausgleichszeugnisse zwischen Besteller und ausführendem Meister", bzw. als „künstlerische Ausgleichserzeugnisse zwischen Kirche und Welt". ${ }^{.58}$

Dass er diese Idee des Ausgleichs nicht allgemein sondern gleichsam im technischen Sinn versteht, erschließt sich aus den zeitgleichen Aufzeichnungen zum Verhältnis von potenzieller und kinetischer Energie in Bezug auf die Darstellung des Pendels. ${ }^{59}$ Wiederholt greift Warburg auf dieses Modell zurück, insbesondere mit Blick auf seine grundlegenden psychologischen Fragestellungen: So nehmen Ausdrücke wie „seelische Schwingung", „Schwingungsweite und Intensität der Schwingung", „Schwingungszustand“, „seelischer Umfang“ und „seelische Dynamik“ auch in späteren Schriften eine fast formelhafte Bedeutung an. ${ }^{60}$

Im Aufsatz über Francesco Sassetti wird bekanntlich die Fortuna als „antikisierendes Energiesymbol der persönlichen Gedankenwelt eines Zeitgenossen Sassettis, des Giovanni Rucellai“ gekennzeichnet, die es ermöglicht,

„Francesco Sassettis Verhältnis zur Antike [...] als natürlichen Gegenpol seiner mittelalterlichen Gesinnung zu begreifen. Denn in seiner sinnbildlichen Verwendung antiker Gebilde offenbaren sowohl Sassetti wie Rucellai, wie sie in jener Übergangsepoche des subjektiven Empfindens einen neuen energetischen Gleichgewichtszustand anstreben, [...] obwohl sie sich des Konfliktes zwischen der Kraft der Einzelpersönlichkeit und rätselhaft zufälliger Schicksalsmacht klar bewußt sind “".61

So werden verschiedene Abbildungen der Fortuna auf die entsprechende Tafel des Bilderatlas Mnemosyne (Abb. 1) wie auf Kraftlinien auf einem Feld disponiert, die diagonal die "Schwingungsweite" vom Mittelalter bis hin zur Hochrenaissance nachzeichnen.

Bezeichnenderweise taucht dasselbe Modell (mit begleitenden Schemata und skizzierten Diagrammen) in den im Sommer 1901 notierten Fragmenten während der intensiven

57 Warburg 1998 , Bd. I.I, S. 95

58 Warburg 1998, Bd. I.1, S. 95 und S. 101

59 Vgl. etwa Warburgs Exzerpt zur potentiellen und kinetischen Energie des Pendels, zitiert von Thomas Hensel: Wie aus der Kunstgeschichte eine Bildwissenschaft wurde. Aby Warburgs Graphien, Berlin 2011, S. 84, Anm. 85 sowie Ulrich Raulff: Wilde Energien. Vier Versuche zu Aby Warburg Göttingen 2003a, bes. S. 137-139. Vgl. auch WarBurg [1927-1929] 2010, S. 643: „Ikonologie des Zwischenraums. Kunsthistorisches Material zu einer Entwicklungsphysiologie des Pendelganges zwischen bildhafter und zeichenmässiger Ursachensetzung".

60 Vgl. etwa Aby Warburg: Bildniskunst und Florentinisches Bürgertum [1902], in WARBURG 1998 Bd. I.1, S. 100, und ders: Orientalisierende Astrologie [1926], in: WARBURG 1998, Bd. I.2, S. 565.

61 WArBURG 1998, Bd. I.1, S. 146 
Arbeit an den Florentiner Aufsätzen auf: „Was zwischen Traum und Tanz liegt ([den] Endpolen des Pendels [Schwingungsweite]) gehört den ,besonnenen Menschen ““ ${ }_{62}$

Vom mechanischen Modell der Pendelschwingung wendet sich Warburg schließlich zum magnetischen Modell einer polaren Spannung: als er den Aufsatz über Sassetti abschließt, spricht er von einer „organische[n] Polarität der weiten Schwingungsfähigkeit eines gebildeten Frührenaissancemenschen, der im Zeitalter der Metamorphose des energetischen Selbstbewußtseins charaktervollen Ausgleich anstrebte “ ${ }^{\text {}}{ }^{63}$

\section{Edgar Wind zwischen Warburg und Cassirer}

Die Bedeutung des grundlegenden Begriffs der Polarität und dessen Nachhall in der Geschichte der Ästhetik wurden von einem der jüngsten geistigen Erben Warburgs mehrmals hervorgehoben: Edgar Wind. Er war es, der insbesondere diesen Aspekt von Warburgs Forschungsansatz - eine komplexe Kombination von philologischer Rekonstruktion und "allgemeinen Ideen" bzw. heuristischen, aus der physikalischen Forschung entlehnten Prinzipien - fortgesetzt hatte, wie im Folgenden anhand einiger Beispiele aus seinen frühen Arbeiten zu zeigen sein wird.

Als er Warburgs Vorgehensweise in einem Text beschrieb, den er wahrscheinlich 1930 für eine Probevorlesung nutzte, schrieb Wind ausgerechnet über den Sassetti-Aufsatz: „In den kulturexegetischen Schriften A. Warburgs ist die bis dahin abstrakt gebliebene Forderung einer ,historischen Psychologie' zu einem wirklichen ,Organon' symbolischer Ausdruckskunde durchgebildet", wobei Warburg „statt einer deskriptiven Typenlehre auf Grund ,innerer Erfahrung - eine polare Ausgleichstheorie der seelischen Energien“ ins Werk gesetzt hatte. ${ }^{64}$

Wind kannte den Text der Fragmente zur Ausdruckskunde, den Warburg ihm zur Lektüre anvertraut hatte, und deren Edition er in den 1930er Jahren für die Institutspublikationen vorbereitete. ${ }^{65}$ Warburg hatte schon früh Winds philosophische Begabung erkannt und geschätzt, ${ }^{66}$ wie etwa aus einem Tagebucheintrag vom 23. September 1928 hervorgeht:

62 Warburg 2011, S. 146, N. 419 (2.VIII.1901); vgl. WarbUrG 2015, S. 214

63 WARBURG 1998 , Bd. I.1. S. 158

64 Edgar Wind: Ưber einige Berührungspunkte zwischen Naturwissenschaft und Geschichte [19301936], in ders.: Das Experiment und die Metaphysik. Zur Auflösung der kosmologischen Antinomien, hg. von Bernhard Buschendorf, Frankfurt am Main 2001, S. 254-269, hier S. 258-260.

65 Obwohl diese Edition nicht realisiert wurde, existieren Dokumente, die Winds Unternehme beweisen: vgl. etwa den Bericht von Fritz Saxl an Erich M. Warburg, datiert auf Februar 193 (Oxford, Bodleian Library, Ms. Wind 240, folder 2), wonach Winds Aufgabe darin bestand, einen Band herauszugeben, , containing the aphorisms in which Warburg put down his notions of philosophy of history and theory of expression to which his historical works have led him ${ }^{\text {c }}$.

66 Vgl. dazu Bernhard Buschendorf; „War ein sehr tüchtiges gegenseitiges Fördern". Edgar Wind und Aby Warburg, in: Idea. Jahrbuch der Hamburger Kunsthalle IV (1985), S. 165-209.

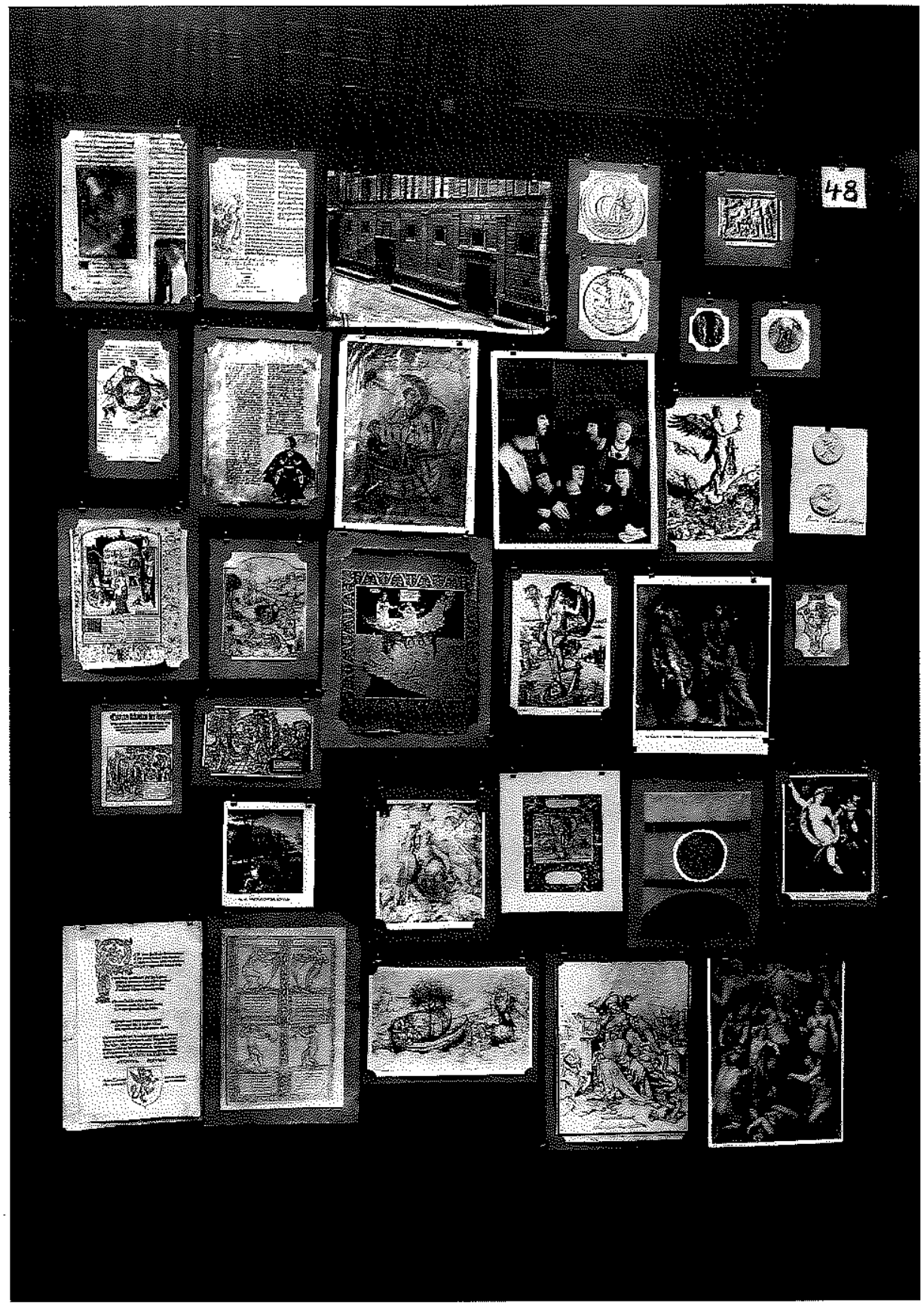

1 Aby Warburg, Tafel 48 des Bilderatlas Mnemosyne, 1929 [aus: WARBURG 2000] 
„Mit Wind ganz generell über den erkenntnistheoretischen Ort der Mnemosyne gesprochen / Physik des Engramms: das sogenannte ,Symbol' erfüllt dabei die Funktion eines \pm energetischen Transformators. $-=$ Hingabe $+=$ Behauptung. Der Zeit geist, magnetisiert[,] der im Phänomen der Gestaltung zu erfassen ist. ,Prolegomena zu einer Physik der seelischen Distanz “ ${ }^{\text {(67 }}$

Wind seinerseits begriff den substantiellen Unterschied, der Warburgs Versuche von den bereits etablierten ikonographischen Studien differenzierte, und konnte das Erklärungspotenzial solcher Versuche (die Rückseite des Teppichs) ausloten.

Überdies weisen bereits Winds frühe Studien ein deutliches Interesse für methodologische und erkenntnistheoretische Fragen auf; das persönliche Treffen mit Warburg mag deshalb möglicherweise eine empfindliche Saite berührt haben, die auch weiter nachklingen sollte.

Mitte der 1920er Jahre, noch vor der persönlichen Bekanntschaft mit Warburg, hatte Wind eine Untersuchung über die naturwissenschaftliche Methode in einem Vortrag skizziert, welche den Nucleus seiner Habilitationsschrift darstellen sollte: Im September 1926, beim Internationalen Kongress der Philosophie in Harvard, sprach er über Experiment and Metaphysics und stellte sich ein Paradoxon als Thema, das der physikalischen Forschung inne wohnt, nämlich: „die Formulierung physikalischer Gesetze gründet sich auf Messungen, die im Gebiet der Physik durchgeführt werden".68 Als Kern dieses circulus vitiosus betrachtete Wind den "Exaktheitsanspruch" der wissenschaftlichen Methode, welche durch eine Analyse der bestimmenden Aspekte von Messungen im experimentellen Verfahren in Frage gestellt werden konnte. Um den circulus vitiosus zu brechen, schlug Wind die Annahme eines metaphysischen Faktors vor, die eine Grundlage für die Ansprüche auf Allgemeingültigkeit der physikalischen Gesetze bilden sollte. Diese Idee, erweitert zu einer "Theorie des Experiments“, sollte zu einem späteren Zeitpunkt ein umfassendes Kapitel der 1934 publizierten Habilitationsschrift bilden. ${ }^{69}$

Als Wind 1936 in einer Festschrift für Ernst Cassirer einen Aufsatz mit dem Titel Some Points of Contact between History and Natural Science publizierte - welcher die englische, teils überarbeitete Version seiner Probevorlesung war ${ }^{70}$ - kehrte er zurück zu seinem auffordernden Argument über die Methodenverwandtschaft zwischen den Naturwissenschaften und der Geschichte: „I shall confine myself to indicating some formal

67 Warburg [1926-1929] 2001, S. 344

68 WIND [1930-1936] 2001, S. 213 (zit. in der Ưbersetzung von Bernhard Buschendorf).

69 Vgl. WIND [1930-1936] 2001, S. 70-121.

70 Edgar Wind: Some Points of Contact between History and Natural Science, in: Raymond Klibansky und Herbert James Paton (Hg.): Philosophy and History. Essays presented to Ernst Cassirer, Oxford 1936, S. 255-264. Unter anderen Abweichungen zwischen der englischen und der ursprünglichen deutschen Fassung dieses Textes scheint es hier interessant hervorzuheben, dass der ausführliche Bezug auf Warburg in der englischen Version nicht mehr vorhanden ist: Vermutlich dachte Wind an eine Leserschaft, die mit den Schriften Warburgs noch nicht vertraut war. points of correspondence between these two worlds - or, to be more precise, between the scientific methods which render each of them an object of human knowledge and experience. The mere assertion that there are such correspondences may appear heretical to many" ${ }^{71}$ Schließlich fuhr er fort mit einem Bezug auf Dilthey, Windelband und Rickert, machte aber auch unmittelbar klar, dass

"the very concept of nature in opposition to which Dilthey proclaimed his Geisteswissenschaft has long been abandoned by the scientists themselves, and the notion of a description of nature which indiscriminately subjects men and their fates like rocks and stones to its ,unalterable laws' survives only as a nightmare of certain historians. Thus it need not be symptomatic of a sinful relapse into the method of thought so generously abused as ,positivistic, if in what follows some examples are chosen to illustrate how the very questions that historians like to look upon as their own are also raised in natural science ${ }^{\text {". }}$.

Wind bezieht sich einerseits auf das Prinzip der Zirkelschlüsse bzw. auf die „Dialektik des historischen Dokuments", das auch in der Quantenmechanik zum Ausdruck kommt: "The scientist is subject to the very same paradox ${ }^{1{ }^{73}}$ Andererseits aber behandelt er die damit verbundene Frage der Nach- oder Rückwirkung der Instrumente bzw. der technischen Neuerungen: „every discovery regarding the objects of their inquiry reacts on the construction of their implements; just as every alteration of the implements makes possible new discovery “. ${ }^{74}$ In seinen abschließenden Betrachtungen verweist Wind auf die „self-transformation of man who has become lord and victim of his own cognition s", und formuliert eine Anregung für die künftige Forschung: „In the study of this self-transformation, scientific and historical research have worked too long independently. It is time that they should be combined" ${ }^{\text {. }}$.

Mit analogen Worten bezeichnete Wind seine Absicht im März 1933 in einem Brief an den Verleger seiner Habilitationsschrift: „die eigentliche Absicht meiner Arbeit [ist], durch eine Analyse der gegenwärtigen Methode der Naturwissenschaften auf Folgerungen (mit Bezug auf den Welt- und Freiheitsbegriff) hinzuweisen, die gerade für die Methode der Geisteswissenschaften von Bedeutung sind “" 7

71 Wind 1936, S. 255. Hier wie in seiner deutschen Fassung der Einleitung zu der Kulturwissenschaft lichen Bibliographie zum Nachleben der Antike bezieht sich Wind auf die von Dilthey, Windelband und Rickert vertretene philosophische Richtung.

72 WIND 1936, S. 256.

73 WIND 1936, S. 257; vgl. S. 261: „The historian disturbs the sleep of the document that he drags forth from a dusty archive. This word, disturbance' is not to be taken as a metaphor, but is meant literally. Even the astronomical physicist acts disturbingly on nature when he splits up a beam of light that has come from the stars, in order to infer the direction and speed of their motion ${ }^{\text {". }}$

74 WIND 1936, S. 262.

75 WIND 1936, S. 264

76 Zit. nach Buschendorfs Vorwort auf WIND [1930-1936] 2001, S.9. 
Ein wesentlicher Bestandteil von Winds Darstellung in seinem Buch über Das Experiment und die Metaphysik ist den Begriffen von "Kausalität und Freiheit ${ }^{477}$ gewidmet, wobei der Autor den kantischen Standpunkt im Licht der Ergebnisse der modernen Physik diskutiert. Wind stützt sich explizit insbesondere auf Max Plancks Problematisierung des Kausalbegriffs in der Physik, ${ }^{78}$ um ein Argument zugunsten der „Vereinbarkeit der menschlichen Freiheit mit der Bindung des Menschen an das Naturgesetz ${ }^{4} \mathrm{zu}$ formulieren. ${ }^{79}$ Eine stetige Sorge um „die gegenwärtige Situation" lässt sich hier klar erspüren, wie auch in einem Brief, den Wind im April 1937 an Ernst Cassirer nach der Lektüre von dessen letzter Monographie, Determinismus und Indeterminismus in der neueren Physik, richtete. Winds Besorgnis, vor allem in Bezug auf das Schlusskapitel des Cassirerschen Werks, lautet: „Sollen wir wirklich zugeben, daß selbst die größten Revolutionen in der Physik ohne Rückwirkung auf die Ethik bleiben? " 80

Neben dem großen Respekt vor dem Philosophen der „symbolischen Formen“ taucht hier wiederum die bittere Kritik politischen Charakters auf, die der junge Wind auch anderswo gegenüber den sogenannten, Vernunftrepublikanern' und ihrer nicht leichten moralischen Verantwortung ausübte.

So erhält der Zusammenhang zwischen Natur- und Geisteswissenschaft bei Wind einen entscheidenden praktischen (und politischen) Akzent; auf erkenntnistheoretischer Ebene hebt er - entsprechend Warburg - methodologische Homologien hervor und fasst sie gleichzeitig in Verbindung mit den Schlussfolgerungen bzw. Rückwirkungen der jeweiligen wissenschaftlichen Entdeckungen, d. h. mit der „Selbstverwandlung des Menschen “ auf.

\section{Niels Bohr am Warburg Institute}

Ein weiteres, weniger bekanntes Beispiel aus Winds organisatorischer Tätigkeit für das Warburg Institute beweist dessen Überzeugung von einem bedeutenden Zusammenhang der Methoden zwischen den Natur- und Geisteswissenschaften. Am 18. Februar 1936 hielt der Physiker und Nobelpreisträger Niels Bohr auf Einladung von Edgar Wind einen Vortrag am Warburg Institute in London über das Thema Some Humanistic Aspects of Natural Science. ${ }^{81}$ Wie aus dem Briefwechsel zwischen Wind, Fritz Saxl und Gertrud Bing vom

77 WIND [1930-1936] 2001, S. 167-204 (3. Kapitel).

78 Wind bezieht sich insbesondere auf Plancks „Rückkehr zum strengen Kausalitätsbegriff durch im Grunde ganz, idealistische Argumente [...]. So stellt Planck ein rein ideelles Reich gedachter Ereignisse, in welchem der Kausalgesetz seine Geltung bewahrt, dem Reich der wirklichen Messungen gegenüber": WiNd [1930-1936] 2001, S. 186, Anm. 71, mit Bezug auf Max Planck: Der Kausalbegriff in der Physik, Leipzig 1932, S. $21 \mathrm{ff}$.

79 Wind [1930-1936] 2001, S. 198.

30 Vgl. Ernst Cassirer: Nachgelassene Manuskripte und Texte, hg. von Klaus Christian Köhnke, John Michael Krois und Oswald Schwemmer, Bd. 18: Ausgewählter wissenschaftlicher Briefwechsel, hg. von John Michaei Krois, Hamburg 2009, S. 172-173, hier S. 172.

81 Vgl. den Einladungsbrief von Edgar Wind an Niels Bohr vom August 1935 (Oxford, Bodleian Library, Ms. Wind 241, Folder 1), den Warburg Institute Annual Report 1934-1935, S. 6-7 (.Forthcoming
Sommer $1935^{82}$ sowie aus dem Einladungsbrief an Bohr hervorgeht, ${ }^{83}$ zielte das Vortragsprogramm für den Winter 1935-36 darauf ab, „die gegenwärtige Lage der Kulturphilosophie von verschiedenen Standpunkten aus [zu] erörtern", und sollte neben den Beiträgen von Historikern und Philosophen auch die Stimme eines Naturwissenschaftlers einschlieBen. ${ }^{84}$ Das Niels Bohr Archiv in Copenhagen verwahrt die Aufzeichnungen, die als Basis für den Londoner Vortrag dienten und den Titel tragen: Some Humanistic Aspects of Atomic Theory. ${ }^{85}$

Nach einigen einleitenden Vorbemerkungen, in denen Bohr sich selbst als „neither scholar nor philosopher" bezeichnete, begann er damit, den „general background of atomic theory" sowie die Zweifel und Widerstände, die gegen sie vorgebracht werden, zu skizzieren. Bei einer Darlegung der jüngst diskutierten Darstellungen der atomaren Struktur zeigte er die Parallelen zwischen Atomstruktur und Solarsystem auf, und definierte die Begriffe der ,Stabilität ${ }^{\iota}$ und ,Komplementarität ${ }^{\prime}$ in der Quantentheorie. Der zentrale Begriff ,Komplementarität ${ }^{856}$ wurde besonders eingehend behandelt, sowohl in Bezug auf

Lectures and Courses") sowie das Vorlesungsverzeichnis des Warburg Institute für das Frühjahr 1936: Warburg Institute lectures, Courses \& Classes January-June 1936, S. [2] (Oxford, Bodleian Library, Ms. Wind 6, Folder 3).

82 In einem Brief an Fritz Saxl vom 3. September 1935 (Oxford, Bodleian Library, Ms. Wind 241, folder 1) schlïgt Wind folsendes Programm vor: Neben einer kleineren Vortragsreihe solt te eine gröBere Ser Bere Serie Wind hoffte auf Bohrs Zusage, weil er als theoretischer Physiker geeigneter als der experimentelle Physiker Rutherford erschien. Bohs positive Antwort (nicht erhalten) kam wenige Tage später, we Wind am 13. September an Saxl mitteilt (Oxford, Bodleian Library, Ms. Wind 24, folder 1).

83 Siehe Anm. 81

84 Neben Bohrs Beitrag sollte der Philosophiehistoriker Francis Macdonald Conford aus Cambridge über „Notwendigkeit und Zufall“ in der antiken Philosophie sprechen, der Neu-Thomist Jacques Maritain über „Science et sagesse“, Anatole De Monzie - der 1935 die Encyclopédie française mitbegründet hatte - über „Die Idee der Encyclopädie“, der Paläograph Elias Avery Lowe über das Thema der kulturellen Oberlieferung, und Ernst Cassirer schließlich sollte „den neukantianer Standpunkt" vertreten. Gemäß Edgar Winds Wünschen hätte das Programm auch einen Vortrag von Paul Valéry enthalten sollen, der aber an einer Teilnahme verhindert war. Das gedruckte Programm der Lectures and Classes des Warburg Institute für die Monate Januar-Juni 1936 weist weitere Abweichungen güber den urprünglichen Absichten auf- Niels Bohrs Vortra fand jedoch tere Abweichungen gegentaber den ursprünglichen Absichten auf, Niels Bohns Vortrag fand jedoch tatsächlich statt. vi. Winds Brief an Saxl vom 2. September 1935 (wie Anm. 82) und den Dankesbrief von Edgar Wind an Niels Bohr vom 21. Januar 1937 (Copenhagen, Niels Bohr Archive (NBA) BGC-WINE-370121).

85 Es handelt sich um zwei von einer fremden Hand in schwarzer Tïnte beschriebene Manuskriptseiten, aufbewahrt im Niels Bohr Archive in Copenhagen unter der Signatur BSM-285-1936. Den auf Englisch notierten Aufzeichnungen sind zwei eigenhändige Anmerkungen mit Bleistift hinzugefügt, die den Anlass („Disposition til Foredrag i Warburg ") und einen synthetischen Verweis auf die nachfolgende Diskussion (einen Bezug auf den Physiker „Rutherford“) registrieren. Dr. Finn Aaserud sei hier für die freundliche Unterstützung herzlich gedankt.

86 Zum ersten Mal wurde der Begriff der ,Komplementarität' Ende der 1920er Jahre formuliert: vgl. Niels Bohr: The Quantum Postulate and the Recent Development of Atomic Theory, in: Nature (Suppl) 121 (1928) S. 580-590 sowie Niels Bohr: Collected Works, ho von Finn Aaserud, Bd 10 Complementarity beyond Physics (1928-1962), hg. von David Farholdt, Amsterdam u. a. 1999. 
das Verhältnis zwischen der Relativitätstheorie und der Quantenmechanik als auch auf die Methoden der biologischen und psychologischen Forschung. Bohr betonte - wie er es auch bei weiteren Gelegenheiten tun sollte, wenn er nicht vor einem Fachpublikum sprach - vor allem die „erkenntnistheoretische Belehrung", welche die neueste Entwicklung der Physik mit sich gebracht hatte. Er definierte einmal die ,Komplementarität' als „eine folgerichtige Verallgemeinerung des Kausalitätsideals ${ }^{\text {“ }}$, g7 die es erlaube, scheinbar widersprüchliche Aufschlüsse, die sich in der Atomphysik über das Verhalten ein und desselben Objekts formulieren lassen, als sich zwar gegenseitig ausschließend, jedoch trotzdem als ,komplementär' zu betrachten.

Damit stellte das Komplementaritätsprinzip den Versuch dar, die Kluft zwischen klassischer und neuer physikalischer Auffassung der Natur zu überbrücken. Mehr noch, es erwies sich in Bohrs Augen als ein viel breiter anwendbares Prinzip, dessen Auswirkungen weit jenseits des Bereichs physikalischer Wissenschaft reichen - bis etwa zur Biologie, Psychologie und Anthropologie, die tiefe epistemologische Affinitäten mit der Physik aufweisen.

In den Aufzeichnungen zum Vortrag gehalten im Warburg Institute findet sich eine Auflistung von Themen, die in diesem Zusammenhang angesprochen wurden: Darunter Iassen sich insbesondere Fragen wie die der „complementary relationships between instinct and reason", der „different situation of animals and humans" und der „independence of human tradition [from] biological inheritance" im Text eines Vortrags weiter verfolgen, den Bohr 1938 beim Internationalen Kongress für Anthropologie und Ethnologie in Kopenhagen hielt. ${ }^{88}$

Bohrs Gedanken zur „Überprüfung und Verfeinerung ${ }^{\text {"89 }}$ von Begriffen, die zwischen den Disziplinen austauschbar sind, erscheinen im Hinblick auf unser Thema besonders subtil und einleuchtend. Bohr konzentrierte sich auf die Bedingungen der Beobachtung und der Beschreibung von Phänomenen sowie auf den Sprachgebrauch überhaupt.

Noch in einem 1954 in New York gehaltenen Vortrag über die „Unity of Knowledge“ fasste er das Problem folgendermaßen zusammen:

„alle Kenntnisse [werden] anfänglich innerhalb eines der Beschreibung früherer Erfahrungen angepaßten begrifflichen Rahmens ausgedrückt und [...] jeder solcher Rahmen [kann sich] mit der Zeit als zu eng erweisen [...], um neue Erfahrungen zu umfassen. [...] Die Ausweitung des begrifflichen Rahmens hat ja nicht nur Ordnung innerhalb der einzelnen Zweige der Wissenschaft geschaffen, sondern auch Ähnlich-

87 Niels Bohr: Erkenntnistheoretische Fragen in der Physik und die menschlichen Kulturen, in: ders.; Atomphysik und menschliche Erkenntnis, Braunschweig 1958b, S. 24.

88 Publiziert mit dem Titel Erkenntnistheoretische Fragen in der Physik und die menschlichen Kulturen, in: BoHr 1958, S. 23-31.

89 Siehe BoHr 1958. keiten unserer Stellung bei der Analyse und Synthese von Erfahrungen innerhalb anscheinend getrennter Wissensgebiete enthüllt und dabei die Möglichkeit einer immer umfassenderen objektiven Beschreibung aufgezeigt"."

Bohrs Schlussfolgerungen zur Frage der Einheit des Wissens können in direkter Verbindung zu seiner Diskussion der Komplementarität im Anschluss an das Problem der vorurteilsfreien Verständigung zwischen verschiedenen menschlichen Kulturen gesehen werden. Beide Argumente, die wohl von der Auswirkung historischer Verhältnisse - der Konsequenzen bzw. der Vorzeichen des zweiten Weltkriegs - geprägt sind, und die im Konferenzraum des kurz zuvor emigrierten Warburg Instituts vorgetragen wurden, standen in Einklang mit dem Konzept selbst des von Warburg gegründeten Forschungsinstituts.

Ein im Niels Bohr Archive aufbewahrter Brief vom Januar 1937 lehrt uns, dass Edgar Wind den dänischen Physiker eingeladen hatte, einen Beitrag für die erste Ausgabe des Journal of the Warburg Institute einzureichen; er bat ihn, das Thema seines Londoner Vortrags auszuwählen oder etwa seine „Anschauungen vom Symbolbegriff“ darzulegen..$^{91} \mathrm{Im}$ Anhang seines Briefs fügte Wind - als Herausgeber der ersten Ausgaben des Journal - eine Beschreibung des Programms der Zeitschrift hinzu, das vermutlich von ihm selbst stammte und stellte fest, dass „das kulturpsychologische Problem der Symbolbildung und -umformung und das geschichtliche Problem des, Nachlebens der Antike die Forschungsrichtung [des Instituts] bezeichnen“. Neben einer historischen Koordinate - die Erforschung des Nachlebens der Antike - sollte gleichwertig eine andere, theoretisch orientierte Achse stehen, um Forschungsgegenstände einer gleichsam stereometrischen Analyse zu unterziehen. In der Erforschung des Symbolproblems kondensierte sich also die Frage nach dem Zusammenhang zwischen Natur- und Geisteswissenschaften.

Auch wenn Bohrs Beitrag nicht im Journal erschien, ist es jedoch möglich, in seinen Aufzeichnungen für den Londoner Vortrag eine klare, mit der so formulierten Forschungsrichtung des Warburg Instituts harmonische Besinnung zu erkennen: „The lesson of physics and especially of atomic theory", liest es sich als letzter Punkt in seinem Manuskript, bestehe aus dem "mutual help of different sciences in the tracing of prejudices". Obwohl mit anderen Forschungszielen und ausgehend von anderen Fragestellungen, setzte sich Warburg mit denselben Themen auseinander, indem er sich vornahm, in seiner "Werkstatt" die „Einheit aller geistigen Orientierungsversuche " zu veranschaulichen.

In dieser Hinsicht stellte nicht zuletzt Warburgs ständige Arbeit am Sprachgebrauch eine weitere tiefe Ähnlichkeit mit Bohrs Reflexionen dar: Seine Assimilation und Ver-

90 Niels Bohr: Einheit des Wissens, in: ders.: Atomphysik und menschliche Erkenntnis, Braunschweig 1958 c, S. 68-69.

91 Edgar Wind an Niels Bohr, 21. Januar 1937 (NBA, BGC-WINE-370121). Bohrs Antwort ist nicht erhalten; im Copenhagener Archiv ist lediglich eine auf den 29. Januar 1937 datierte Nachricht aufbewahrt (NBA, BGC-WINE-370129), die Bohrs Selcretärin an Wind richtete, um ihm mitzuteiten, 
wandlung von Begrifflichkeiten aus dem Gebiet der Physik könnte als ein komplexer Versuch interpretiert werden, die ,Beschreibungsinstrumente ${ }^{\epsilon}$ des Kunst- und Kultur historikers zu überprüfen und zu verfeinern, seine wissenschaftliche Sprache vor den Verlegenheiten des alltäglichen Sprachgebrauchs sowie der poetisch anmutenden Sprache der ästhetisierenden Kunstgeschichte zu wahren und sie gleichsam zu sterilisieren'. Eine anspruchsvolle Aufgabe, die sich nur von der ,Rückseite des von Warburg in seinen publizierten Schriften geduldig geknüpften Gewebes her auswerten lässt.

Im Anschluss an Warburgs Arbeit an den ,Beschreibungsinstrumenten' und ,-bedingungen' des Kunst- und Kulturhistorikers stehen Edgar Winds Überlegungen zu den Zirkelschlüssen und der Dialektik des historischen Dokuments, welche wiederum die ,Beobachtungsbedingungen' thematisieren: Ein Anliegen, das sich wie ein roter Faden durch Winds gesamte Forschung hindurch zieht. Im ersten Entwurf der für seine 1958 gesammelten Aufsätze Pagan Mysteries in the Renaissance verfassten „Bemerkung zur Methode“ schrieb Wind:

"Once we get rid of the prejudice that the best document for interpreting a painting is one that duplicates it in words, the documents which seem to evade an answer become remarkably communicative. [...] When we have reached this point, that a picture helps us to place the right accents in a text, and a text to place the right accents in a picture, they will both acquire a new luminosity: and this is all we should aim for ${ }^{\ll}$. 92

Anstelle der Suche nach Eins-zu-eins-Beziehungen von Bild und Text betonte Wind was man ihre ,Komplementarität' nennen könnte.

Dass dabei offensichtlich ein Zusammenhang bzw. eine Ähnlichkeit zwischen den Natur- und Geisteswissenschaften in rein erkenntnistheoretischer Hinsicht besteht und nicht eine ,Eins-zu-eins-Beziehung', brachte Wind schlagkräftig zum Ausdruck, indem er seine methodologischen Überlegungen mit der grundlegenden Frage der Logik verband:

"a method of demonstration is required which is radically different from mathematical proofs. In the place of a linear logic, in which each proposition has its well-defined antecedents by which it is linked to a well-defined set of premises, we must aim for a configurational logic by which contingent arguments are interlocked “. 93

In diesen Sätzen wird dieselbe Aufgabe bzw. Herausforderung formuliert, die Niels Bohr seinerseits für das Durchsetzen des neuen Paradigmas der Quantenphysik erkannte, als er schrieb, dass die Erklärung der mit der neuen Theorie einhergehenden scheinbaren

22 Edgar Wind, Picture and Text, in: ders.: The Religious Symbolism of Michelangelo. The Sistine Ceiling, hg. von Elisabeth Sears, Oxford 2000, S. 192-193.

93 WIND 2000, S. 193.
Widersprüche „nur durch eine genauere Untersuchung der Grenzen gefunden werden [könnte], die die neuen Erfahrungen selber dem unzweideutigen Gebrauch der in die Beschreibung der Phänomene eingehenden Begriffe auferlegen".. 


\section{NATURBILDER}

IMAGES OF NATURE

Herausgegeben von

Frank Fehrenbach, Robert Felfe und Iris Wenderholm

BAND 6

\section{ABY WARBURG UND DIE NATUR \\ Epistemik, Ästhetik, Kulturtheorie}

Herausgegeben von Frank Fehrenbach und Cornelia Zumbusch 
Gedruckt mit großzügiger Unterstützung durch die Stiftung Universität Hamburg und die Alexander von Humboldt-Stiftung

ISBN 978-3-11-037445-2

eISBN (PDF) 978-3-11-058341-0

Library of Congress Control Number: 2019938537

Bibliografische Information der Deutschen Nationalbibliothek

Die Deutsche Nationalbibliothek verzeichnet diese Publikation in der Deutschen Nationalbibliografie;

detaillierte bibliografische Daten sind in Internet über http://dnb.dnb.de abrufbar.

(c) 2019 Walter de Gruyter GmbH, Berlin/Boston

Einbandabbildung: Sandro Botticelli, Ankunft der Venus, um 1490. Florenz, Uffizien (Detail)

Reihenlayout und Satz: Petra Florath, Stralsund

Druck und Bindung: Beltz Grafische Betriebe GmbH, Bad Langensalza

www.degruyter.com

\section{Inhaltsverzeichnis}

Frank Fehrenbach und Cornelia Zumbusch

Aby Warburg und die Natur.

Zur Einleitung

Kurt W. Forster

Warburgs Mneme nach einer langen Pause des Vergessens

Giovanna Targia

Der "Zusammenhang zwischen Natur- und Geisteswissenschaft "

bei Aby Warburg, Edgar Wind und Niels Bohr

Hans Christian Hönes

Spielraum der Rationalität.

Warburg und die Wahrscheinlichkeitsrechnung

Stefan Rieger

,Causale Virtualität

Energie und Übertragung bei Warburg

Michael Neumann

"Bilderwirthschaft".

Über die epistemologische Verselbständigung kultureller Semantiken

Philipp Ekardt

Bewegungsimpressionen nach der Natur - Warburg mit Vignoli

Caroline van Eck

„Du lebst und thust mir nichts"!

Fear, empathy and projection 
Aby Warburg und die Natur - diese Zusammenstellung könnte auf den ersten Blick überraschen, scheint Warburg als Sammler von Pathosformeln und Theoretiker eines abendIändischen Bildgedächtnisses doch eher auf den Menschen, dessen Gebärdensprache und die sich darin organisierenden psychischen Energien fixiert gewesen zu sein. Warburgs Interesse am bewegten Leben der antiken Kulte, der frühneuzeitlichen Feste oder Umzüge und den daraus hervorgehenden Bildprägungen führt ihn tatsächlich vor allem auf die mimischen und gestischen Ausdrucksmöglichkeiten des menschlichen Körpers. Die antike Mänade und die Ninfa fiorentina, der sterbende Orpheus und der von Schlangen umwundene Laokoon, Verfolgungs- und Entführungsszenen zeigen allesamt tanzende, schreitende, kämpfende oder ringende Körper. Wenn Warburg diese Pathosformeln als Ausdrucksbewegungen bezeichnet, die er im Mnemosyne-Atlas zur einer Grammatik der Gebärdensprache zusammenstellen will, dann steht im Mittelpunkt seines Interesses der menschliche Körper und dessen Ausdrucksmöglichkeiten, die sich zu einer Kultur- und Bildgeschichte fügen sollen. Agon und Todesangst, Verzückung, Ekstase und Raserei, Lähmuing und Depression prägen das ,Seelendrama ${ }^{a_{1}}$ des Abendlands, das sich in der Bildenden Kunst von der Antike bis zu Neuzeit zeigt.

Stellt man die Optik allerdings von den scharf umrissenen Konturen bewegter Körper auf deren Umgebungen um, dann geraten andere Bewegungsformen in den Blick. Bereit in seiner 1893 erschienenen Dissertation über Botticellis Geburt der Venus verschiebt Warburg den Blick von den mythologischen Figuren auf ihre Haare und Gewänder, die er als Transmissionsriemen der Affektbewegung beschreibt. Georges Didi-Hubermann hat hie

* Unser großer Dank für die sorgfältige editorische Ưberarbeitung der Texte und die Erstellung der Gesamtbibliographie geht an Antonia Goetz, Laura Wittwer, Daniel Mayr, Katharina Stutz und Antonina Tetzlaff.

1 Vgl. dazu: Ilsebill Barta-Fliedl: Vom Triumph zum Seelendrama. Suchen und Finden oder Die Abentheuer eines Denklustigen, in: dies. und Christoph Geissmar (Hg.): Die Beredsamkeit des Leibes. Zur Körpersprache in der Kunst, Salzburg/Wien 1992, S. 165-170; Dorothée Bauerle: Gespenstergeschichte für ganz Erwachsene. Ein Kommentar zu Aby Warburgs Bilderatlas Mnemosyne, Münster 1988 . 